\title{
Effect of Ni Interlayer on Cavitation Erosion Resistance of NiTi Cladding by Tungsten Inert Gas (TIG) Surfacing Process
}

\author{
Zhen-Ping Shi ${ }^{1,2}$. Zheng-Bin Wang ${ }^{1} \cdot J^{\text {J-Qiang Wang }}{ }^{1} \cdot$ Yan-Xin Qiao $^{3} \cdot$ Huai-Ning Chen ${ }^{1}$ - Tian-Ying Xiong ${ }^{1}$. \\ Yu-Gui Zheng ${ }^{1}$
}

Received: 17 April 2019 / Revised: 3 July 2019 / Published online: 10 October 2019

(C) The Chinese Society for Metals (CSM) and Springer-Verlag GmbH Germany, part of Springer Nature 2019

\begin{abstract}
The NiTi cladding with/without Ni interlayer was prepared on stainless steel (SS) by tungsten inert gas (TIG) surfacing process, aiming at achieving good cavitation erosion resistance. The ranking according to the cavitation erosion resistance is NiTi plate $>$ NiTi-Ni-TIG cladding $>$ NiTi-TIG cladding $>$ SS. The better cavitation erosion resistance of NiTi-TIG and NiTi-Ni-TIG claddings than SS substrate is due to their higher micro-hardness and superelasticity. Furthermore, the existence of $\mathrm{Ni}$ interlayer can decrease the amount of brittle intermetallic compounds, such as $\mathrm{Fe}_{2} \mathrm{Ti}$, and inhibit the crack generation, which results in the higher cavitation erosion resistance of NiTi-Ni-TIG compared with NiTi-TIG cladding. Thus, the cavitation erosion resistance of NiTi cladding prepared by TIG surfacing process can be improved by employing Ni interlayer.
\end{abstract}

Keywords Cavitation erosion $\cdot \mathrm{NiTi} \cdot$ Cladding $\cdot$ Tungsten inert gas surfacing process $\cdot \mathrm{Ni}$ interlayer

\section{Introduction}

Cavitation erosion often occurs in flowing and ultrasonic systems where the static pressure is below the vapor pressure of liquid [1, 2]. In general, the vapor bubbles can form when the pressure is above the vapor pressure, and the subsequent collapse of the unstable bubbles can create shock wave or micro-jet in turbulent flows, which can impact surfaces and induce cavitation erosion damage [1,3-5]. Therefore, many resources will be used for repairing the damaged components caused by cavitation erosion every year $[6,7]$.

Available online at http://link.springer.com/journal/40195

Zheng-Bin Wang

zbwang12s@imr.ac.cn

Yan-Xin Qiao

yxqiao@just.edu.cn

1 CAS Key Laboratory of Nuclear Materials and Safety Assessment, Institute of Metal Research, Chinese Academy of Sciences, Shenyang 110016, China

2 School of Materials Science and Engineering, University of Science and Technology of China, Shenyang 110016, China

3 School of Materials Science and Engineering, Jiangsu University of Science and Technology, Zhenjiang 212003, China
The near equiatomic NiTi alloy has superior properties, such as superelasticity [8], high corrosion resistance [9], high fatigue strength [10], and biocompatibility [11], in particular excellent cavitation erosion (CE) resistance [9]. These properties correlate with the austenite phase B2 $[12,13]$. Cheng et al. [14] found that the cavitation erosion property of $\mathrm{NiTi}$ alloy was related to its superelasticity rather than hardness. The superelasticity of the NiTi alloy can mitigate the impact stress induced by cavitation erosion and prevent the crack propagation. Therefore, NiTi alloy is regarded as a promising candidate for cavitation-erosion-resistant material.

However, due to the inferior weldability and high price, NiTi alloy has been limited in the application in cavitation erosion environment [15]. In order to overcome its shortcomings, many preparation technologies have been used to develop NiTi coating against cavitation erosion, such as low-pressure plasma spraying (LPPS) [3], air plasma spraying (APS) [16], high velocity oxy fuel spraying (HVOF) [17], laser spraying [18, 19], sputter depositing [20] and cold spraying [21]. Unfortunately, these coatings do not exhibit excellent cavitation erosion resistance due to the pores in the coatings [22, 23]. The authors [24] investigated the cavitation erosion behavior of NiTi coating produced by APS from the pre-alloyed NiTi powder and found that the pores in NiTi coating would become a cavitation source during 
the cavitation erosion process. Alternatively, the dense NiTi claddings prepared by welding may reduce the pores and acquire the excellent cavitation erosion resistance. Richman et al. [25] reported that both the martensitic and austenitic NiTi alloys explosively welded to steel exhibited excellent cavitation erosion resistance. However, explosive welding is generally used for the components with flat or coaxial cylindrical surfaces, which restricts its application [26]. Cheng et al. [27] found that NiTi cladding prepared by microwaveassisted brazing showed high cavitation erosion resistance but lower corrosion resistance compared with $316 \mathrm{~L} \mathrm{SS}$. Wu et al. [11] found that the NiTi cladding produced by infrared brazing had high cavitation erosion resistance compared with the SUS304 SS. Similar results were reported by Yang et al. [12], and they found that NiTi thin film produced by filtered arc deposition system (FADS) exhibited superior cavitation erosion resistance compared with 316 SS. Cheng et al. [28] also prepared the NiTi cladding on AISI 316 SS by laser welding. However, the equipment and running costs of laser welding limit its application [29, 30].

The tungsten inert gas (TIG) is a cheap and convenient industrial process, which is applicable in the factories or laboratories [31, 32]. The TIG process needs no chamber and can be used on the surface with more complicated geometry. Nevertheless, some cracks and brittle intermetallic compounds, such as $\mathrm{Fe}_{2} \mathrm{Ti}$, were formed in the joints when NiTi alloy was welded to stainless steel by TIG process [33]. The Ni interlayer was added to improve the weldability, such as laser welding [33, 34] and friction welding [35]. The $\mathrm{Ni}$ interlayer can decrease the brittle intermetallic compounds and inhibit the crack generation [33, 34]. However, the work on the influence of $\mathrm{Ni}$ interlayer on the TIG processed NiTi cladding is rarely reported. In the present work, NiTi cladding on SS with/without Ni interlayer was prepared by TIG surfacing process. The microstructure and cavitation erosion behavior of NiTi cladding were investigated.

\section{Experimental Details}

\subsection{Materials and Sample Preparation}

NiTi wire containing austenite B2 and stainless steel (SS) plate were used in this investigation, whose compositions are listed in Table 1. The dimension of NiTi wire is $100 \mathrm{~mm}$ in length, $2 \mathrm{~mm}$ in diameter. The SS was selected as the substrate with $140 \mathrm{~mm}$ in length, $50 \mathrm{~mm}$ in width and $5 \mathrm{~mm}$ in thickness, which was ground by grinding wheel before TIG surfacing process. The pure Ni wire was used as the interlayer material, and its composition is given in Table 1 . The dimension of the Ni wire was $90 \mathrm{~mm}$ in length and $1.8 \mathrm{~mm}$ in diameter. Before TIG surfacing process, the oxides on the surface of NiTi and Ni wires were removed by grinding, and then, the wires were cleaned in an alcohol bath and dried in air. In addition, the NiTi alloy plate containing austenite B2 was used as the comparing material.

\subsection{TIG Surfacing Process}

In the TIG surfacing process (Master MLS 3500, KEMPPI, Southern Finland, Finland), deposition of NiTi or Ni was carried out manually, with an operating current of $100 \mathrm{~A}$, an operation voltage of $10 \mathrm{~V}$ and argon flowing of $10 \mathrm{~L} / \mathrm{min}$ as the shielding gas. The NiTi claddings with/without $\mathrm{Ni}$ interlayer were designated as NiTi-Ni-TIG and NiTi-TIG claddings, respectively. Before the deposition of $\mathrm{NiTi}$ on $\mathrm{Ni}$ for the NiTi-Ni-TIG cladding, the oxides on the Ni layer were removed by grinding.

\subsection{Microstructures}

The claddings and NiTi plate were mounted in epoxy, then ground from $120^{\#}$ to $2000^{\#}$ using a series of silicon carbide abrasive papers and finally polished using 1 - and $0.25-\mu \mathrm{m}$ diamond polishing pastes. The microstructure and morphology of claddings were observed by the optical microscope (OM) (Carl Zeiss Axio Observer Z1 m, ZEISS, Oberkochen, Germany) and the FEI XL30 field emission gun scanning electron microscope (FEG-SEM, FEI, and Hillsboro, USA). Energy-dispersive spectroscopy (EDS) analyses were also performed to clarify the compositions of claddings. X-ray diffraction (XRD) using a Righaku D/max 2400 diffractometer (Rigaku Corporation, Tokyo, Japan) with monochromated $\mathrm{Cu} k \alpha$ radiation $(\lambda=0.1542 \mathrm{~nm})$ was used to characterize the phase composition.

\subsection{Indentation Test}

The micro-hardnesses of the NiTi-Ni-TIG cladding, NiTi-TIG cladding, NiTi plate and SS were measured
Table 1 Chemical compositions of Ni wire and SS (wt \%)

\begin{tabular}{lllllllllll}
\hline & $\mathrm{C}$ & $\mathrm{Si}$ & $\mathrm{Mn}$ & $\mathrm{P}$ & $\mathrm{S}$ & $\mathrm{Cr}$ & $\mathrm{Ni}$ & $\mathrm{Fe}$ & $\mathrm{Ti}$ & $\mathrm{Al}$ \\
\hline SS & 0.041 & 0.52 & 7.93 & 0.044 & 0.005 & 14.69 & 4.17 & $\mathrm{Bal}$ & - & - \\
Ni wire & $\leq 0.15$ & $\leq 0.7$ & $\leq 1.0$ & $\leq 0.02$ & $\leq 0.015$ & - & $\mathrm{Bal}$ & $\leq 1.0$ & $2.0-3.5$ & $\leq 1.5$ \\
NiTi wire & - & - & - & - & - & - & 55.57 & - & 44.43 & - \\
NiTi plate & - & - & - & - & - & - & 55.57 & - & 44.43 & - \\
\hline
\end{tabular}


using a HV-1000 micro-hardness tester (Laizhou hengyi tester equipment co., Ltd, Laizhou city, Shandong province, China). The Vickers micro-hardness on the surface or along the depth of the cross section was measured at a load of $500 \mathrm{~g}$ and a loading time of $10 \mathrm{~s}$. Nano-indentation tests were commonly employed to study the superelasticity, a property closely related to the cavitation erosion resistance [36]. The nano-indentation characteristic of the NiTi-Ni-TIG cladding, NiTi-TIG cladding, NiTi plate and SS was acquired using a nano-indentation tester (Tribo-Indenter, Hysitron, USA), with the maximum load of $30 \mathrm{mN}$ and the loading time of $10 \mathrm{~s}$. The surface elastic behavior of the sample can be assessed by the depth recovery ratio $\eta_{\mathrm{h}}$ defined as:

$\eta_{\mathrm{h}}=\frac{h_{\max }-h_{\mathrm{r}}}{h_{\max }}$,

where $h_{\max }$ is the maximum penetration depth and $h_{\mathrm{r}}$ is the residual depth after unloading [14]. The energy recovery ratio $\eta_{\mathrm{w}}$ is defined as:

$\eta_{\mathrm{w}}=\frac{W_{\mathrm{rc}}}{W_{\mathrm{t}}}=\frac{\int_{h_{r}}^{h_{\max }} F \mathrm{~d} h}{\int_{0}^{h \max } F \mathrm{~d} h} V$

where $W_{\mathrm{rc}}$ and $W_{\mathrm{t}}$ are the reversible and total work done, respectively. Liu et al. [37] reported that the superelasticity of the sample was evaluated by the ratio $\eta_{\mathrm{w}}$. A material with higher $\eta_{\mathrm{w}}$ exhibits more elasticity/superelasticity has greater capabilities to absorb impact energy and can bear the deformation with less damage.

\subsection{Cavitation Erosion Test}

The cavitation erosion test was conducted using an ultrasonic vibratory apparatus (53 Church Hill Rd., Newtown, CT, USA) according to the ASTM G32-10 (2010) standard [38]. The schematic diagram of the equipment is shown in Fig. 1. The vibration frequency of the probe was $20 \mathrm{kHz}$, and the peak-to-peak amplitude was $60 \mu \mathrm{m}$. Before the cavitation erosion test, the sample was ground to $1000^{\#}$ using a series of silicon carbide abrasive papers, washed in the alcohol, dried in hot air, and weighted using an analytical balance (AUY220, Shimadzu, Japan) with an accuracy of $0.1 \mathrm{mg}$. During the cavitation erosion test, the sample was submerged into the distilled water and was placed at a distance of $0.5 \mathrm{~mm}$ to the lower surface of the probe that was immersed into the test medium to a depth of $15 \mathrm{~mm}$. The temperature of the distilled water was maintained at about $20 \pm 1{ }^{\circ} \mathrm{C}$ using the cooling water. Each test was repeated at least three times to ensure the reproducibility. The sample was weighed at regular time intervals, and the weight loss was converted to a mean depth of cavitation erosion (MDE) according to [2]:

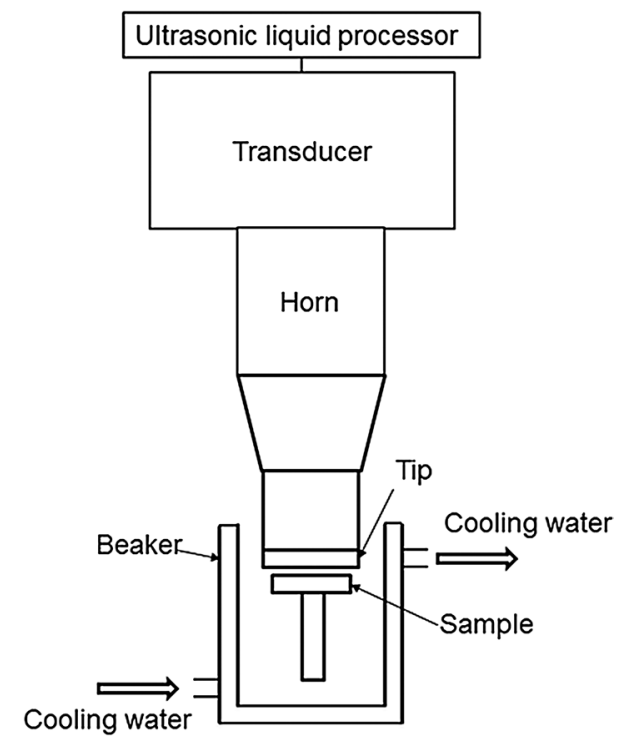

Fig. 1 Schematic diagram of the cavitation erosion tester

$\operatorname{MDE}(\mu \mathrm{m})=\frac{10 \Delta W}{\rho A}$,

$\Delta W=\left(\Delta W_{1}+\Delta W_{2}+\Delta W_{3}\right) / 3$,

where $\Delta W$ is the mean weight loss in $\mathrm{mg}, \Delta W_{i}$ is the weight loss of the sample in $\mathrm{mg}, A$ is the eroded area in $\mathrm{cm}^{2}$ and $\rho$ is the density of the surface layer in $\mathrm{g} / \mathrm{cm}^{3}$.

\section{Results and Discussion}

\subsection{Composition, Microstructure and Micro-hardness}

\subsubsection{NiTi-TIG Cladding}

The cross-sectional view of NiTi-TIG cladding is shown in Fig. 2. The NiTi-TIG cladding consists of NiTi coating, interface zone and substrate, and the interface is metallurgical bond. Small amounts of pores can be observed, and it is noticed that there are also some through-wall cracks in the NiTi-TIG cladding. This type of crack is frequently observed in the NiTi/SS dissimilar welding joints using plasma [34, 38], laser [39], resistance [40] and friction [35] welding, which can release the residual stress during the TIG process [41].

Figure 3 shows the SEM microstructure, the EDS elements line distribution and the micro-hardness profiles in the cross section of NiTi-TIG cladding. The EDS scanning was performed on the cross section of the sample in Fig. 3a. Three regions of coating, interface and substrate based on 


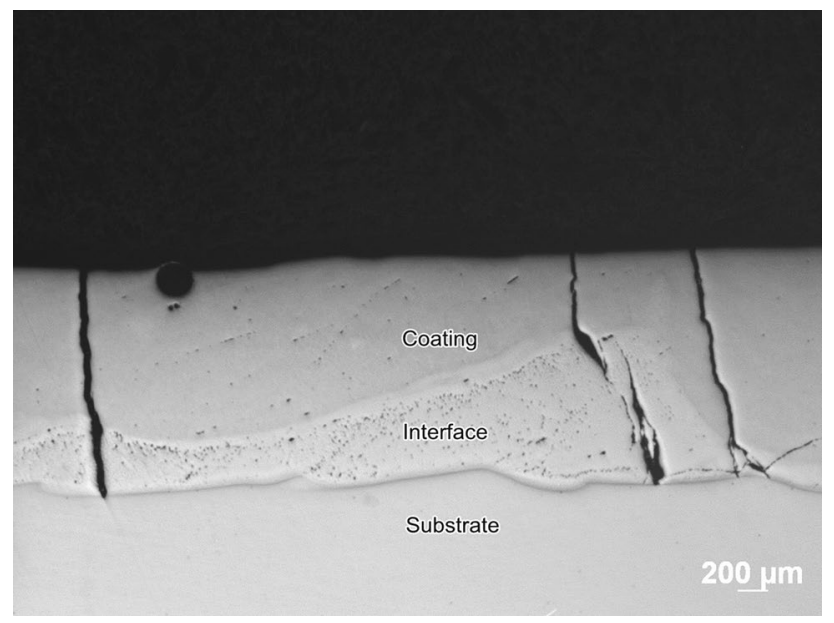

Fig. 2 Cross-sectional view of NiTi-TIG cladding

the chemical composition content are shown in Fig. 3b. The element fluctuation in the interface zone is more evident than both in the coating and substrate. The contents of $\mathrm{Cr}, \mathrm{Mn}$ and $\mathrm{Fe}$ decrease while those of $\mathrm{Ni}$ and $\mathrm{Ti}$ increase from the substrate to coating. In Fig. 3c, the micro-hardness of coating is similar to that of interface zone (about $945 \mathrm{HV}_{500}$ ), and they are both higher than those of the substrate (about $403 \mathrm{HV}_{500}$ ), NiTi plate (about $307 \mathrm{HV}_{500}$ ) and NiTi wire (about $281 \mathrm{HV}_{500}$ ).

The XRD patterns of NiTi-TIG cladding are shown in Fig. 4. The characteristic peaks of $\mathrm{Fe}_{2} \mathrm{Ti}, \mathrm{B} 2, \gamma(\mathrm{Fe}, \mathrm{Ni})$, $\mathrm{Ni}_{3} \mathrm{Ti}_{1} \mathrm{Ni}_{4} \mathrm{Ti}_{3}$ and $\mathrm{Ni}$ can be identified. The $\mathrm{Fe}_{2} \mathrm{Ti}$ is the brittle phase, the presence of which could deteriorate the cavitation erosion resistance of the cladding.

\subsubsection{NiTi-Ni-TIG Cladding}

Figure 5 shows a cross-sectional view of NiTi-Ni-TIG cladding. It consists of the NiTi coating, Ni interlayer and substrate, and the interface of each layer is metallurgical bond. There are small pores shown in Fig. 5, which are similar to those in Fig. 2. In fact, the quantities of the pores in NiTi-TIG cladding and NiTi-Ni-TIG cladding are little. In contrast to NiTi-TIG cladding (Fig. 2), there are no cracks
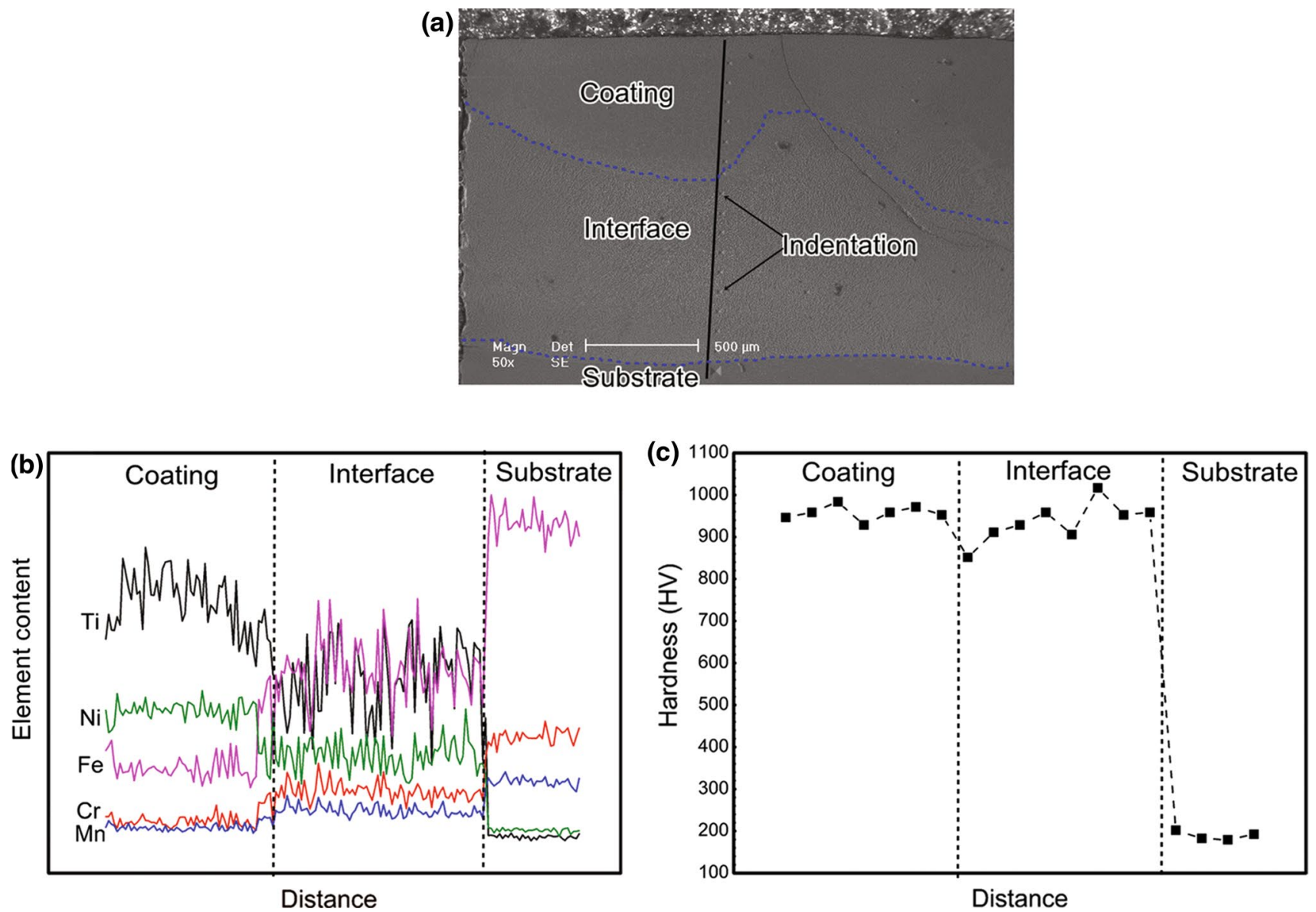

Fig. 3 a SEM microstructure, b distribution of elements from substrate to coating by EDS, $\mathbf{c}$ micro-hardness profiles in the cross section of NiTi-TIG cladding 


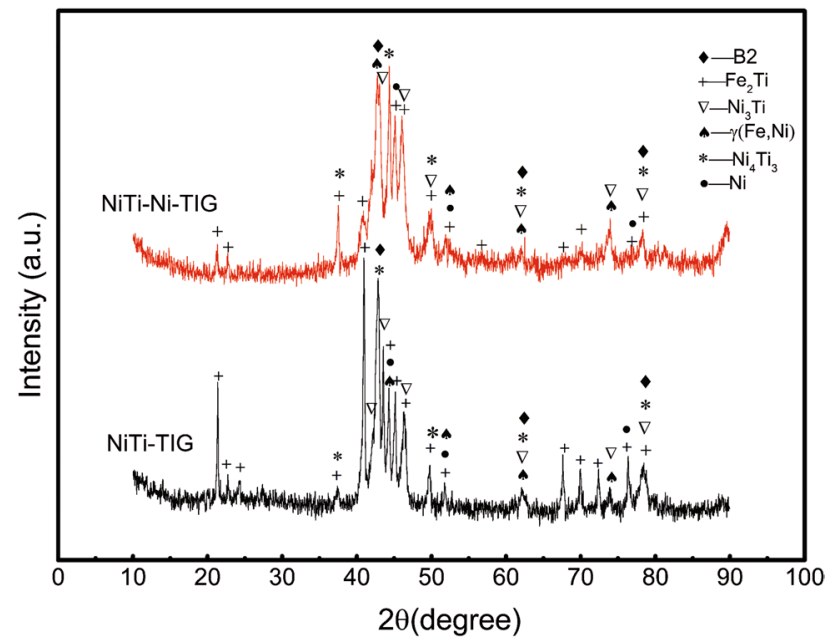

Fig. 4 XRD pattern of NiTi-TIG cladding and NiTi-Ni-TIG cladding

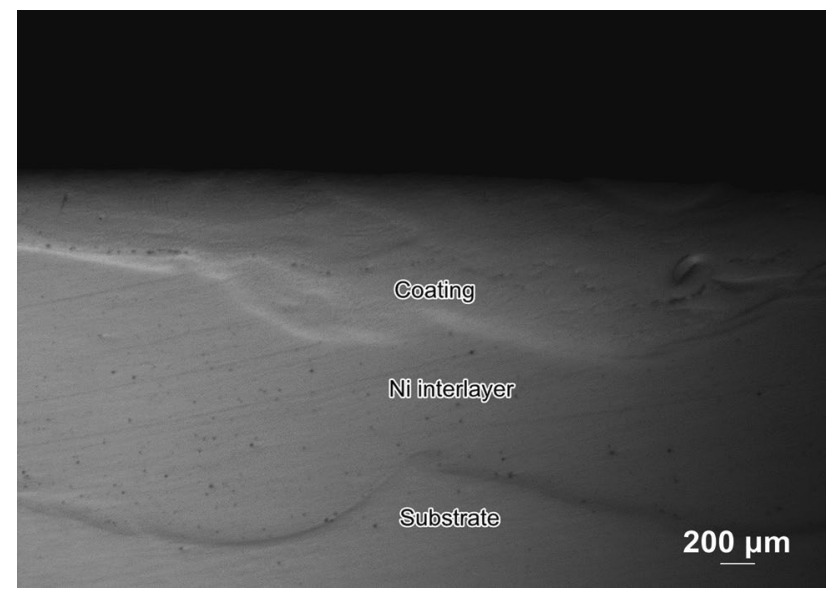

Fig. 5 Cross-sectional view of NiTi-Ni-TIG cladding

in the NiTi-Ni-TIG cladding due to the addition of Ni interlayer. The linear expand coefficient of $\mathrm{Ni}$ is between $\mathrm{NiTi}$ and stainless steel, which is beneficial to release the residual stress generated during the TIG surfacing process. Therefore, Ni interlayer can inhibit the crack generation [33, 34].

Figure 6a shows the SEM microstructure in the cross section of NiTi-Ni-TIG cladding. Figure 6b, c displays the distribution of chemical compositions and micro-hardness along the black line in Fig. 6a from the substrate to the coating. The NiTi-Ni-TIG cladding can be divided into three layers of NiTi coating, Ni interlayer and substrate according to the chemical composition shown in Fig. 6b, which is consistent with Fig. 5. The contents of $\mathrm{Cr}, \mathrm{Mn}$ and $\mathrm{Fe}$ decrease while those of $\mathrm{Ni}$ and $\mathrm{Ti}$ increase from the substrate to coating. It is worthy of noting that the content of $\mathrm{Fe}$ in the coating layer is higher compared with the NiTi-TIG cladding (Fig. 3b), indicating that the Ni interlayer does not act as a diffusion barrier. Meanwhile, the content of $\mathrm{Ni}$ in the coating layer is higher than that of $\mathrm{Ti}$, which is quite different from the corresponding content relationship in NiTi-TIG cladding (Fig. 3b). The difference of $\mathrm{Fe}$ and $\mathrm{Ni}$ contents between NiTi-TIG cladding and NiTi-Ni-TIG cladding should account for the reduction of brittle phase $\mathrm{Fe}_{2} \mathrm{Ti}$ after adding the Ni interlayer, which will be discussed later. Besides, the Vickers indentation in the $\mathrm{Ni}$ interlayer (Fig. 6a) shows no signs of cracking, indicating that the Ni interlayer is tough [42]. In Fig. 6c, the micro-hardnesses of the coating, Ni interlayer and the substrate is $610 \mathrm{HV}_{500}, 141$ $\mathrm{HV}_{500}$ and $190 \mathrm{HV}_{500}$, respectively. The micro-hardness of NiTi-Ni-TIG cladding coating is higher than those of $\mathrm{Ni}$ interlayer and substrate.

The micro-hardnesses of NiTi-TIG cladding and NiTi-Ni-TIG cladding are higher than those of SS and NiTi wire. It is mainly attributed to the formation of the brittle phases $\left(\mathrm{Fe}_{2} \mathrm{Ti}\right)$. The brittle phases are formed in NiTi side because NiTi has a lower melting point than SS. In addition, the lattice distortions caused by residual stresses induced by the thermal gradient can also contribute to the hardness increase [43]. Nevertheless, the micro-hardness of NiTi-Ni-TIG cladding is lower than that of NiTi-TIG cladding. It should be attributed to the lower content of brittle phase $\mathrm{Fe}_{2} \mathrm{Ti}$, which can be confirmed by the lower intensity of the diffraction peak of $\mathrm{Fe}_{2} \mathrm{Ti}$ in the XRD pattern presented in Fig. 4. Some investigations also reported that the adding $\mathrm{Ni}$ is favorable to eliminate the formation of brittle phase $\mathrm{Fe}_{2} \mathrm{Ti}[33,34]$. The reduction of $\mathrm{Fe}_{2} \mathrm{Ti}$ content should not be related to the effect of Ni interlayer on Fe content, because of that Fig. 6b shows higher Fe content in the coating. Instead, it is attributed to the reduction of the fusion ratio of base metals (NiTi and SS) by adding the Ni interlayer [33, 34].

Based on the analyses above, it can be concluded that the main microstructure difference between NiTi-TIG cladding and NiTi-Ni-TIG cladding is the number of cracks and brittle phases. The addition of $\mathrm{Ni}$ interlayer can eliminate the cracks and inhibit the formation of brittle phases. The former results from the stress release effects of $\mathrm{Ni}$ interlayer as mentioned above, while the latter is due to the dilution of $\mathrm{Ni}$.

\subsection{Indentation Properties}

The cavitation erosion resistance of NiTi alloy has been deeply related to the elastic behavior that can be assessed by the depth recovery ratio $\eta_{\mathrm{h}}$ and the energy recovery ratio $\eta_{\mathrm{w}}$ in indentation tests $[14,37]$. Figure 7 shows that the force-displacement curves during loading and unloading cycles acquired in nano-indentation tests. The nanoindentation characteristics of NiTi-TIG and NiTi-Ni-TIG claddings are similar to each other. The TIG-deposited NiTi claddings exhibit higher hardness and smaller maximum displacement compared with NiTi plate and SS substrate. 
(a)
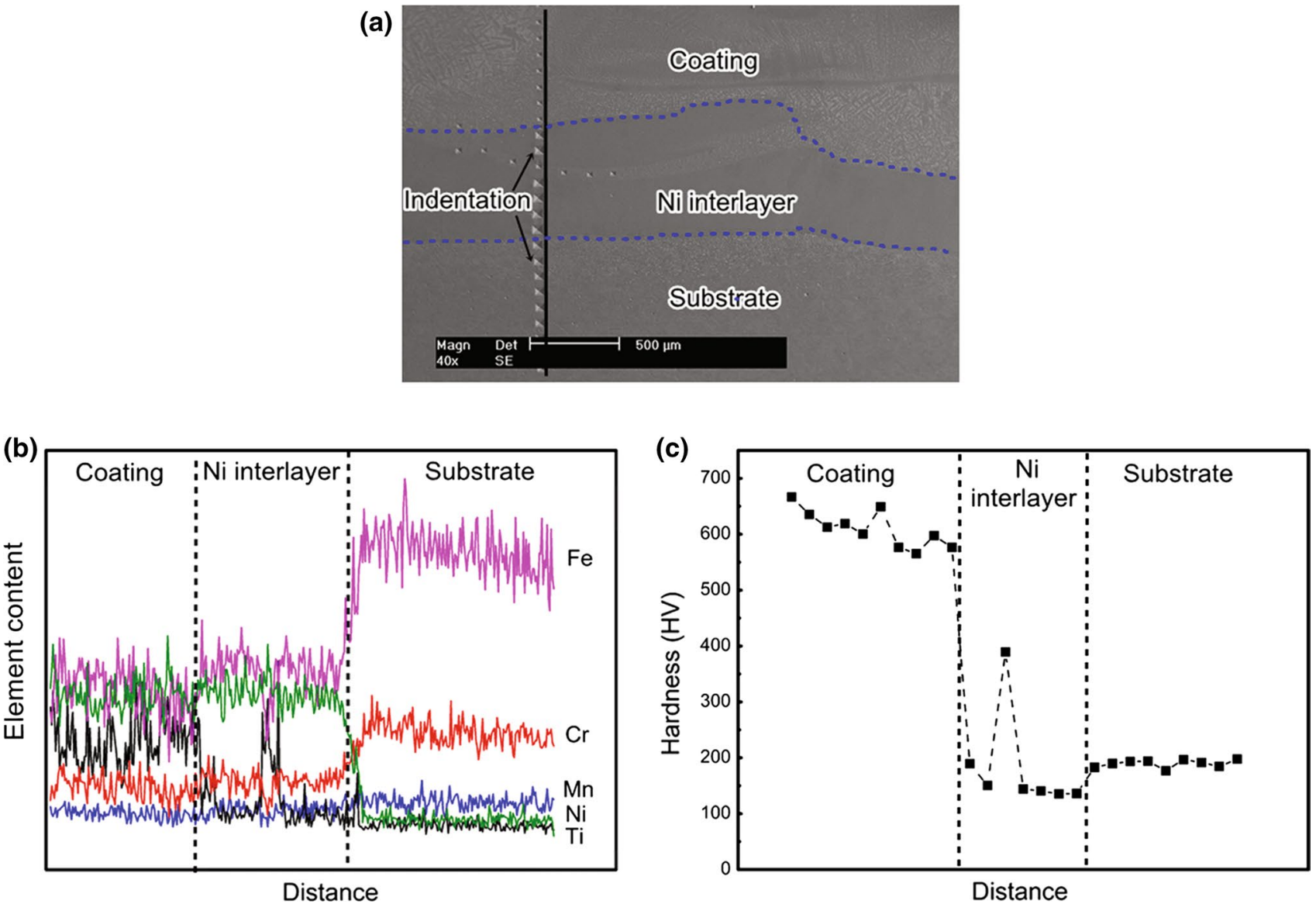

Fig. 6 a SEM microstructure, b distribution of elements from substrate to coating by EDS, $\mathbf{c}$ micro-hardness profiles in the cross section of NiTi-Ni-TIG cladding

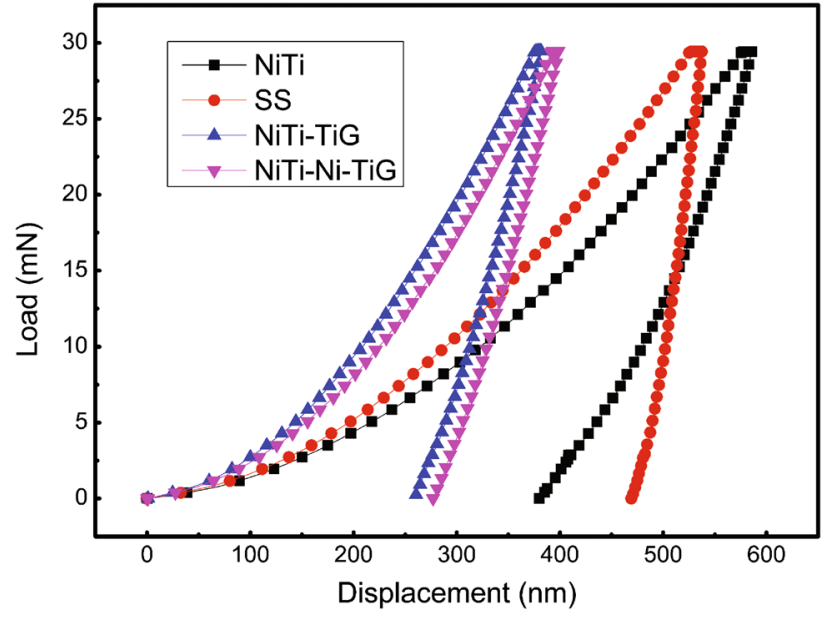

Fig. 7 Force-displacement curves in nano-indentation tests

Based on Eqs. (1) and (2), the depth recovery ratio $\eta_{\mathrm{h}}$ and the energy recovery ratio $\eta_{\mathrm{w}}$ of the four tested materials are listed in Table 2 . The depth recovery ratio $\eta_{\mathrm{h}}$ and the energy recovery ratio $\eta_{\mathrm{w}}$ of NiTi plate and TIG-deposited NiTi
Table 2 Properties of various types of samples

\begin{tabular}{lcll}
\hline Sample & $\begin{array}{l}\text { Maximum dis- } \\
\text { placement }(\mathrm{nm})\end{array}$ & $\eta_{\mathrm{h}}$ & $\eta_{\mathrm{w}}$ \\
\hline SS & $536.75 \pm 20.26$ & $0.129 \pm 0.014$ & $0.138 \pm 0.014$ \\
NiTi-TIG & $385.26 \pm 10.96$ & $0.324 \pm 0.014$ & $0.389 \pm 0.008$ \\
NiTi-Ni-TIG & $390.30 \pm 10.61$ & $0.303 \pm 0.013$ & $0.368 \pm 0.012$ \\
NiTi plate & $600.29 \pm 18.98$ & $0.302 \pm 0.019$ & $0.346 \pm 0.013$ \\
\hline
\end{tabular}

claddings are similar, demonstrating similar superelasticity. It indicates that the addition of $\mathrm{Ni}$ interlayer has no effects on the superelasticity. The superelasticity of NiTi alloy mainly depends on the $\mathrm{B} 2$ phase and the $\mathrm{B} 2$ phase is the main phase for both NiTi-TIG cladding and NiTi-Ni-TIG cladding. This is the exact reason why the superelasticity of these two cladding is similar to each other. In contrast, $\eta_{\mathrm{h}}$ and $\eta_{\mathrm{w}}$ of the TIG-deposited NiTi claddings and NiTi plate are much higher than that of the SS substrate. Cheng et al. also found similar results in NiTi cladding [28]. Accordingly, it can be concluded that the superelasticities of TIGdeposited NiTi claddings and NiTi plate are superior to that 
of SS substrate. The higher hardness and more superelasticity should probably result in the better cavitation erosion resistance of NiTi-TIG cladding and NiTi-Ni-TIG cladding compared with SS.

\subsection{Cavitation Erosion Behavior}

Figure 8 shows the mass losses with cavitation time and MDE of SS, NiTi-TIG cladding, NiTi-Ni-TIG cladding and NiTi plate. It can be found from Fig. 8a that there are no obvious mass losses for all samples before the first $2 \mathrm{~h}$, and this period is usually referred as the incubation period. After the incubation period, the mass loss of SS increases rapidly, especially after $5 \mathrm{~h}$. In contrast, the NiTi plate is still in the incubation time with little mass loss. The rank of cavitation erosion resistance for tested materials is $\mathrm{NiTi}>\mathrm{NiTi}-\mathrm{Ni}$-TIG cladding $>$ NiTi-TIG cladding $>$ SS. Based on Fig. 8, the cumulative mass loss of NiTi-TIG cladding after $11 \mathrm{~h}$ test is 2.6 times as much as that of NiTi-Ni-TIG cladding. Some authors believe that the difference in cumulative mass loss at this level is noticeable $[9,11,18]$.

Figure 9 shows the damage morphologies of the SS, NiTi plate, NiTi-TIG cladding and NiTi-Ni-TIG cladding after cavitation erosion for $11 \mathrm{~h}$. The SS exhibits a deeply eroded surface as shown in Fig. 9a. The inserted image in Fig. 9a shows a typical ductile fracture mode. For the NiTi plate, some small cavitation pits distribute evenly on the surface and the dimension of these pits varies in size but is no more than $10 \mu \mathrm{m}$, as shown in Fig. 9b. The cavitation behavior of NiTi-Ni-TIG cladding is similar to that of NiTi plate except that the number and size of cavitation pits are greater, as shown in Fig. 9d.

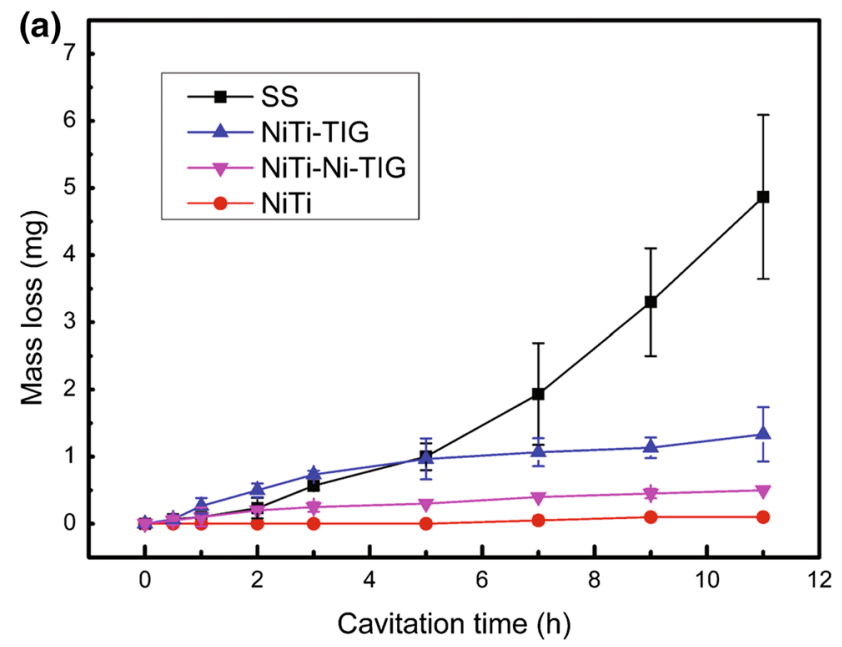

Figure 9c shows the damage morphologies of the NiTi-TIG cladding. The surface damage of NiTi-TIG cladding is not uniform, and there are mainly two kinds of damaged area (area A and B), except the undamaged area C. This is consistent with the results of Cheng et al. [31] who also found similar morphology that both the slightly damaged region and the severely damaged region existed on the surface of NiTi-TIG cladding after cavitation erosion. It is obvious that the mass loss of area A concentrates on the region at the vicinity of cracks, while a typical brittle fracture mode of erosion is clearly visible on area B in Fig. 9c.

The chemical compositions of area $\mathrm{B}$ and area $\mathrm{C}$ in Fig. $9 \mathrm{c}$ are listed in Table 3. The content of $\mathrm{Fe}$ at the undamaged area (area C) is much lower than that of severely damaged area (area B). The Mn could be ignored because of its little content. According to the $\mathrm{Cr}-\mathrm{Ti}-\mathrm{Fe}$ ternary phase diagram [44], the solubility of $\mathrm{Cr}$ is high in $\mathrm{Fe}_{2} \mathrm{Ti}$ (> 60 at $\%$ ). The phases of area $\mathrm{B}$ and area $\mathrm{C}$ can be predicted based on the isothermal section of the $\mathrm{Fe}-\mathrm{Ni}-\mathrm{Ti}$ ternary system at $1000{ }^{\circ} \mathrm{C}$ [45]. The reason for selecting $1000{ }^{\circ} \mathrm{C}$ isothermal section of the Fe-Ni-Ti ternary system is that the high cooling rate does not allow sufficient time for atomic diffusion during cooling. Therefore, the phases formed during the heating stage are likely to be retained at room temperature, regardless of the influence of cooling [34]. Miranda et al. $[34,46]$ also used the $1000{ }^{\circ} \mathrm{C}$ isothermal section of the $\mathrm{Fe}-\mathrm{Ni}-\mathrm{Ti}$ ternary system to determine the composition of the phase in the dissimilar welding of NiTi to SS. Accordingly, the phases of area $\mathrm{B}$ and area $\mathrm{C}$ may be predicted to be $\mathrm{Fe}_{2} \mathrm{Ti}$ and $\mathrm{B} 2$, respectively. Hence, the brittle phase $\mathrm{Fe}_{2} \mathrm{Ti}$ is damaged severely under cavitation attack while B2 with superelasticity is still intact. In addition, quite few severely damaged areas around the pores were found, which could be attributed to the small amount. It suggests that the pores are

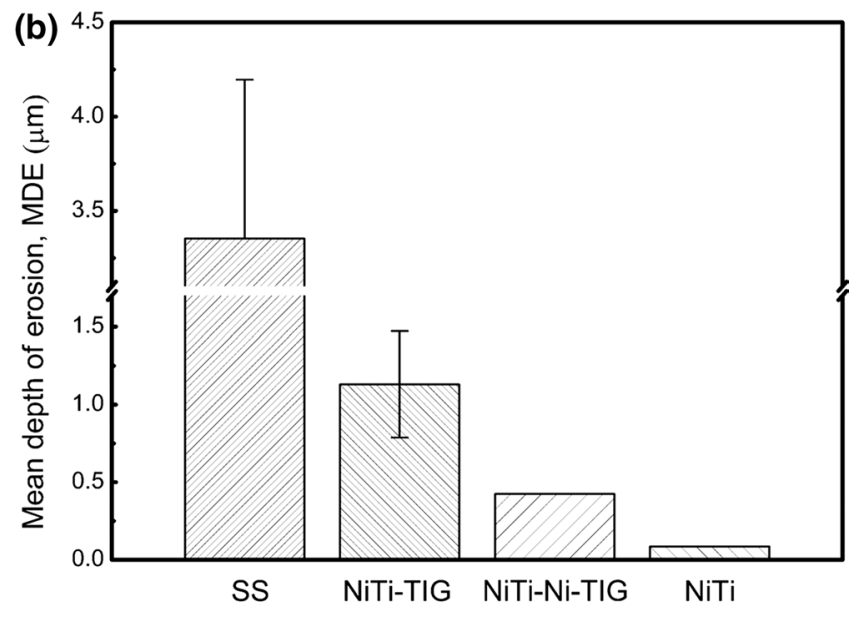

Fig. 8 Comparison of cavitation erosion behavior of SS, NiTi-TIG cladding, NiTi-Ni-TIG cladding and NiTi plate: a erosion curves, $\mathbf{b}$ cumulate mean depth of erosion (MDE) after cavitation erosion for $11 \mathrm{~h}$ 
(a)

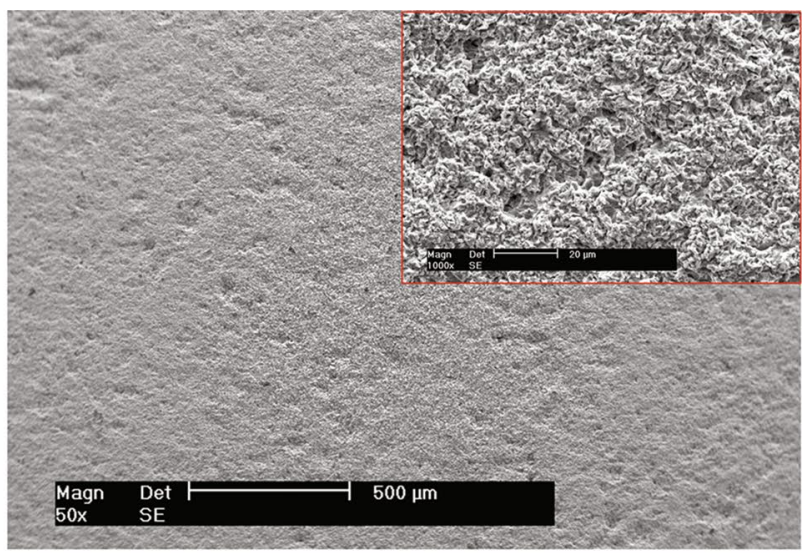

(c)

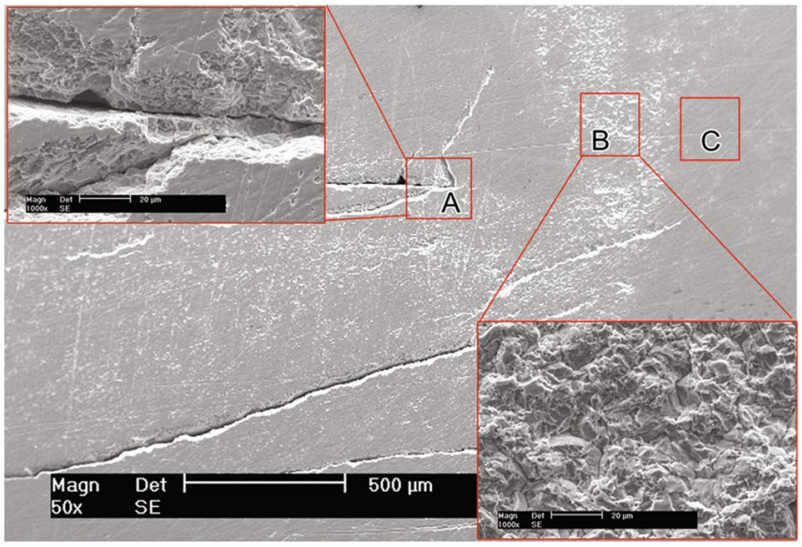

(b)

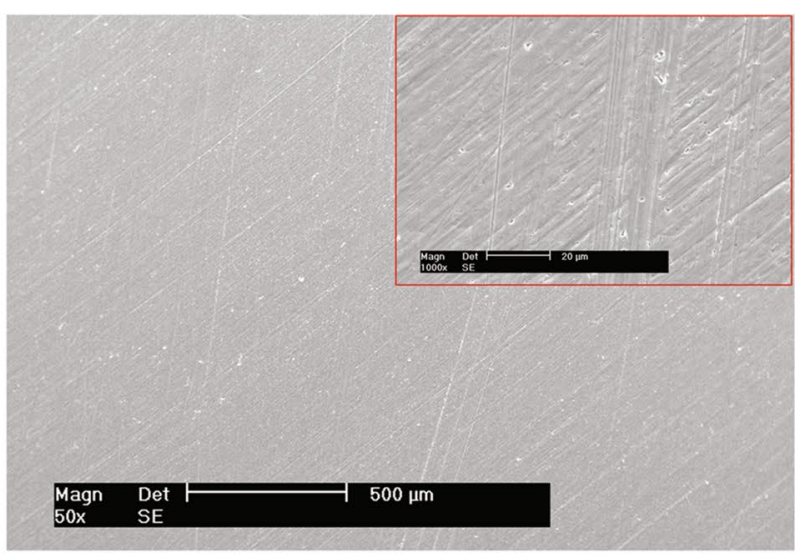

(d)

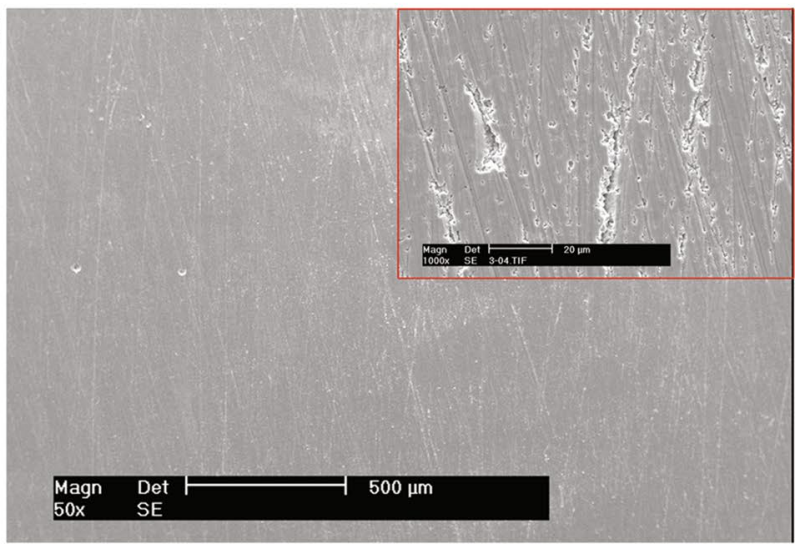

Fig. 9 SEM images after cavitation erosion for 11 h. a SS; b NiTi plate; $\mathbf{c}$ NiTi-TIG cladding; $\mathbf{d}$ NiTi-Ni-TIG cladding

Table 3 Chemical compositions of the selected areas from NiTiTIG cladding in Fig. 9c

\begin{tabular}{llllrll}
\hline Selected area & \multicolumn{2}{l}{ Compositions (at\%) } & \multicolumn{2}{c}{ Potential phases } \\
\cline { 2 - 5 } & $\mathrm{Ti}$ & $\mathrm{Cr}$ & $\mathrm{Mn}$ & $\mathrm{Fe}$ & $\mathrm{Ni}$ & \\
\hline $\mathrm{B}$ & 28.19 & 8.14 & 4.00 & 34.53 & 25.14 & $\mathrm{~B} 2$ \\
$\mathrm{C}$ & 41.17 & 2.39 & 1.27 & 9.97 & 45.20 & $\mathrm{Fe}_{2} \mathrm{Ti}$ \\
\hline
\end{tabular}

not the main factor that deteriorates the cavitation erosion resistance.

Compared with TIG-deposited NiTi cladding, the SS exhibits poorer cavitation erosion resistance due to its low hardness and low recovery ratio of the surface. The NiTi plate shows the highest cavitation erosion resistance due to the superelasticity. During the cavitation erosion test, the fatigue crack tips can also be retarded and stabilized due to stress relief by the superelasticity [11]. Even though the superelasticity and micro-hardness of TIG-deposited NiTi cladding are equal to and higher than that of NiTi plate, respectively, the TIG-deposited NiTi cladding shows relatively lower cavitation erosion resistance. Some second phases or cracks existing in the TIG-deposited NiTi cladding should be the reasons. In the NiTi-Ni-TIG cladding, there are some second phases such as the $\mathrm{Ni}_{3} \mathrm{Ti}, \mathrm{Ni}, \gamma(\mathrm{Fe}$,
$\mathrm{Ni}$, $\mathrm{Fe}_{2} \mathrm{Ti}$ and $\mathrm{Ni}_{4} \mathrm{Ti}_{3}$ as shown in Fig. 4, which do not have superelasticity. In the NiTi-TIG cladding, except these second phases, there are also some cracks. These second phases and cracks can decrease the cavitation erosion resistance of NiTi-TIG cladding. Accordingly, it can be concluded that it is the second phases or cracks formed during the TIG process, rather than the increased micro-hardness, affect the cavitation erosion resistance of TIG-deposited NiTi cladding.

Compared with the NiTi-TIG cladding, the NiTi-Ni-TIG cladding has better cavitation erosion resistance. It should result from the Ni interlayer in the following two aspects. The first is that the Ni interlayer can inhibit the crack generation during cooling. This is confirmed by surface and cross-sectional observation. Figure $9 \mathrm{c}$ shows that the cracks in NiTi-TIG cladding can be the cavitation source during the 
cavitation erosion. Bubble formation can be enhanced near the cracks. Thus, severe micro-jet and/or shock wave can impact the area near the cracks preferentially. Stress concentration at the boundaries of the cracks could also contribute to a more severe attack. The second is that the Ni interlayer can decrease the content of brittle phase $\mathrm{Fe}_{2} \mathrm{Ti}$. The brittle phase $\mathrm{Fe}_{2} \mathrm{Ti}$ is easy to fracture and peel off during the cavitation erosion compared with the B2 phase. Figure $9 \mathrm{c}$ shows that the severely damaged area in NiTi-TIG cladding contains more brittle phases such as $\mathrm{Fe}_{2} \mathrm{Ti}$ and the undamaged area contains more B2 with superelasticity. Accordingly, to reduce the brittle phases and cracks formed during the TIG process should be significant for the improvement of cavitation erosion resistance of TIG-NiTi cladding, which can be fulfilled by employing the $\mathrm{Ni}$ interlayer.

\section{Conclusions}

The NiTi claddings (NiTi-TIG and NiTi-Ni-TIG) on stainless steel (SS) by TIG surfacing process are successfully manufactured. The following conclusions are drawn.

1. The micro-hardness and superelasticity of NiTi claddings are higher than SS substrate, which results in superior cavitation erosion resistance of NiTi claddings. In comparison, the NiTi claddings exhibit inferior cavitation erosion resistance than NiTi plate due to the existence of cracks and brittle phases.

2. The addition of Ni interlayer in NiTi-TIG cladding can eliminate the cracks by releasing the residual stress generated in the TIG surfacing process and inhibit the formation of brittle phases by the dilution effect of adding Ni interlayer, while it has few effects on the superelasticity. Accordingly, the cavitation erosion resistance of NiTi-Ni-TIG cladding is enhanced compared with NiTi-TIG cladding.

Acknowledgements The authors would like to acknowledge Hang Liang and Zhaoxuan Zhang for preparing NiTi cladding by TIG surfacing process. This work was supported by the Opening Project of Material Corrosion and Protection Key Laboratory of Sichuan Province (No. 2017CL18) and The Strategic Priority Research Program of the Chinese Academy of Sciences (Grant Number XDA13040500).

\section{References}

[1] B.K. Sreedhar, S.K. Albert, A.B. Pandit, Wear 372-373, 196 (2017)

[2] Y.X. Qiao, Z.H. Tian, X. Cai, J. Chen, Y.X. Wang, Q.N. Song, Tribol. Lett. 67, 9 (2018)

[3] Y.X. Qiao, S. Wang, B. Liu, Y.G. Zheng, H.B. Li, Z.H. Jiang, Acta Metall. Sin. 52, 240 (2016)
[4] M. Bitzer, N. Rauhut, G. Mauer, M. Bram, R. Vassen, H.P. Buchkremer, Wear 328, 377 (2015)

[5] Y.X. Qiao, X. Cai, J. Cui, Y.B. Tang, H.B. Li, Z.H. Jiang, Mater. Tehnol. 51, 938 (2017)

[6] J.S. Carlton, Marine Propellers and Propulsion, 1st edn. (Butterworth Heinemann, Oxford, 1994), p. 199

[7] Q.N. Song, N. Xu, Y.F. Bao, J.W. Gu, Y.G. Zheng, Y.X. Qiao, Acta Metall. Sin. (Engl. Lett.) 30, 720 (2017)

[8] Y.N. Zhao, S.Y. Jiang, Y.Q. Zhang, Acta Metall. Sin. (Engl. Lett.) 30, 770 (2017)

[9] R.H. Richman, Wear 157, 407 (1992)

[10] I. Kaya, H. Tobe, H.E. Karaca, Acta Metall. Sin. (Engl. Lett.) 29, 286 (2016)

[11] S.K. Wu, H.C. Lin, C.H. Yeh, Wear 244, 93 (2000)

[12] L.M. Yang, A.K. Tieu, D.P. Dunne, S.W. Huang, H.J. Li, D. Wexler, Wear 267, 243 (2009)

[13] V. Stolyarov, Acta Metall. Sin. (Engl. Lett.) 31, 1310 (2018)

[14] F.T. Cheng, P. Shi, H.C. Man, Scr. Mater. 45, 1089 (2001)

[15] C. Elahinia, Shape Memory Alloy Actuators: Design, Fabrication, and Experimental Evaluation, 6th edn. (Wiley, Ltd, 2016), pp. 191-199

[16] M.M. Verdian, K. Raeissi, M. Salehi, Corros. Sci. 52, 1059 (2010)

[17] M.M. Verdian, K. Raeissi, M. Salehi, J. Alloys Compd. 507, 46 (2010)

[18] H. Hitoshi, I. Takashi, M. Akira, S. Hirofumi, Wear 231, 278 (1999)

[19] H. Hitoshi, I. Takashi, S. Hirofumi, Surf. Coat. Technol. 139, 100 (2001)

[20] X. Zhang, J.H. Sui, Y.C. Lei, W. Cai, Acta Metall. Sin. (Engl. Lett.) 30, 1235 (2017)

[21] S. Tria, O. Elkedim, R. Hamzaoui, X. Guo, F. Bernard, N. Millot, Powder Technol. 210, 188 (2011)

[22] C. DeCrescenzo, D. Karatza, D. Musmarra, S. Chianese, T. Baxevanis, P.T. Dalla, Adv. Mater. Sci. Eng. 2018, 10 (2018)

[23] C.J. Huang, X.C. Yan, W.Y. Li, W.B. Wang, C. Verdy, M.P. Planche, Appl. Surf. Sci. 451, 66 (2018)

[24] Z.P. Shi, J.Q. Wang, Z.B. Wang, Y.X. Qiao, T.Y. Xiong, Y.G. Zheng, Coatings 8, 346 (2018)

[25] R.H. Richman, Wear 181-183, 85 (1992)

[26] T.T. Zhang, W.X. Wang, J. Zhou, Acta Metall. Sin. (Engl. Lett.) 30, 991 (2017)

[27] K.Y. Chiu, F.T. Cheng, H.C. Man, Mater. Sci. Eng., A 407, 281 (2005)

[28] F.T. Cheng, K.H. Lo, H.C. Man, Mater. Sci. Eng., A 380, 29 (2004)

[29] Z. Zeng, B. Panton, J.P. Oliveira, A. Han, Y.N. Zhou, Smart Mater. Struct. 24, 125036 (2015)

[30] J.P. Oliveira, B. Panton, Z. Zeng, C.M. Andrei, Y. Zhou, R.M. Miranda, F.M. BrazFernandes, Acta Mater. 105, 15 (2016)

[31] F.T. Cheng, K.H. Lo, H.C. Man, Surf. Coat. Technol. 172, 315 (2003)

[32] D.K. Zhang, G.Q. Wang, A.P. Wu, Acta Metall. Sin. (Engl. Lett.) 32, 694 (2019)

[33] H.M. Li, D.Q. Sun, X.L. Cai, P. Dong, W.Q. Wang, Mater. Des. 39, 293 (2012)

[34] J. Pouquet, R.M. Miranda, L. Quintino, S. Williams, Int. J. Adv. Manuf. Technol. 61, 212 (2011)

[35] S. Fukumoto, T. Inoue, S. Mizuno, K. Okita, T. Tomita, A. Yamamoto, Sci. Technol. Weld. Join. 15, 130 (2013)

[36] Y.X. Qiao, J. Chen, H.L. Zhou, Y.X. Wang, Q.N. Song, H.B. Li, Z.B. Zheng, Wear 424-425, 77 (2019)

[37] R. Liu, D.Y. Li, Mater. Sci. Technol. 16, 332 (2013)

[38] ASTM G32-10 Standard Test Method for Cavitation Erosion Using Vibratory Apparatus; ASTM International: West Conshohocken, PA, USA, 2010 
[39] H. Li, D. Sun, X. Cai, P. Dong, X. Gu, Opt. Laser Technol. 45, 460 (2013)

[40] Q. Li, Y. Zhu, J. Guo, J. Mater. Process. Technol. 249, 548 (2017)

[41] C. van der Eijk, H. Fostervoll, Z.K. Sallom, O.M. Akselsen, Plasma welding of NiTi to NiTi, stainless steel and Hastelloy C276. Join. Adv. Spec. Mater. 6, 129 (2004)

[42] G.R. Anstis, P. Chantikul, B.R. Lawn, D.B. Marshall, J. Am. Ceram. Soc. 64, 538 (1981)
[43] J.R. Weng, J.T. Chang, K.C. Chen, J.L. He, Wear 255, 224 (2003)

[44] S. Wang, K. Wang, G. Chen, Z. Li, Z. Qin, X. Lu, Calphad. 56, 168 (2017)

[45] G. Cacciamani, K.J. De, R. Ferro, U.E. Klotz, J. Lacaze, P. Wollants, Intermetallics 14, 1325 (2006)

[46] J.S. Kirkaldy, L.C. Brown, Can. Metall. Q. 2, 115 (1963) 